\title{
Observation of Axial Phase Evolution of Highly Confined Light Fields
}

\author{
Myun-Sik Kim, ${ }^{1, *}$ Toralf Scharf, ${ }^{1}$ Stefan Mühlig, ${ }^{2}$ Carsten Rockstuhl, ${ }^{2}$ \\ and Hans Peter Herzig ${ }^{1}$ \\ ${ }^{1}$ Optics \& Photonics Technology Laboratory, Ecole Polytechnique Fédérale de Lausanne (EPFL) Breguet 2, 2000 Neuchâtel, \\ Switzerland \\ ${ }^{2}$ Institute of Condensed Matter Theory and Solid State Optics, Abbe Center of Photonics, Friedrich-Schiller-Universität Jena, \\ 07743 Jena, Germany \\ *Corresponding author: myunsik.kim@epfl.ch
}

\begin{abstract}
Highly confined light fields demonstrate peculiar phase features, e.g., Gouy phase anomaly. Longitudinal-differential phase measurements by a high-resolution interference microscope are applied to investigate phase evolutions of confined light fields for physical optical phenomena. OCIS Codes: (260.6042) Singular optics; (290.4020) Mie theory; (180.3170) Interference microscopy.
\end{abstract}

\section{Introduction}

The Gouy phase anomaly is the axial phase shift observed for a convergent optical beam when measuring along the optical axis through a focus. It is named in honor of Gouy's discorvery [1-3]. In his original experiments, the light diverging from a point source was reflected from a planar and a concave mirror and the two beams from each mirror were overlapped in order to form interference fringes. In such a situation circular fringes appear and the central fringe was found to change from dark to bright, or vice verse, at observation planes just before or after the focal point. This change of intensity indicates a $\pi$ phase shift when passing through the focus [3]. Numerous observations of such a phase anomaly in different types of focused beams have been reported, for example, in non-diffracting Bessel beams [4], in Laguerre-Gaussian (LG) beams [5], and radially polarized beams [6]. In addition to the highly focused optical beams, surface plasmon-polaritons [7] and matter waves [8] also exhibit such a phase anomaly.

In this work, we experimentally investigate the axial phase shift for two particular physical optics experiments: the scattered hotspot that is termed photonic nanojet [9] and a diffractive hotspot that is well-known as the spot of Arago [3,10].

\section{Experimental setup}

For our study we employ the high-resolution interference microscope (HRIM) to measure amplitude and phase in the entire three-dimensional (3D) space. The details of the experimental setup are reported in our previous works [11-14]. All experimental and theoretical investigations were performed at a single wavelength of $642 \mathrm{~nm}$ (CrystaLaser: DL640-050-3). Since measurements of amplitude fields are subject to the diffraction limit of the observation system, we use a high numerical aperture objective 100X/NA0.9 (Leica Microsystems, HC PL FLUOTAR) in order to obtain high resolution. The HRIM allows us to measure the longitudinal-differential phase compared to a propagating plane wave by scanning the sample. The plane wave is considered as a constant during such axial scans.

For the photonic nanojet, microspheres are made of borosilicate glass and purchased from Duke Scientific Inc. Their refractive index is 1.55 at $642 \mathrm{~nm}$ and their diameter is $2 \mu \mathrm{m}$. Spheres are dispersedly deposited on the glass substrate and well isolated. For the spot of Arago, a Cr disc of diameter $4 \mu \mathrm{m}$ and thickness $150 \mathrm{~nm}$ is employed and forms a well-defined circular obstacle.

\section{Results and discussions}

The measured phase distribution in the $x-z$ plane of the photonic nanojet is shown in Fig. 1(a). The dark circle indicates the sphere. Outside of the sphere it is assumed that a plane wave propagats without perturbation. The axial phase profiles from measurements and simulations are shown in Fig. 1(b). Simulations are made by Mie-scattering theory and Fourier optics. The axial phase evolution relative to the propagating plane wave traveling beside the sphere, demonstrates a shift of about $\pi$ when passing through the point of peak intensity. For the first time the existence of the Gouy phase anomaly in such scattered hotspots could be confirmed [14]. In our presentation we will discuss more details of the experiment.

The spot of Arago or Poisson's bright spot is the canonical proof of the wave nature of light [see Chapter 18 of Ref. 3]. A bright spot is formed by constructive interference of diffracted light from the rim of the circular obstacle behind an opaque obstacle. Due to the fact that such diffracted light travels other optical path compared to the propagating plane wave along the $\mathrm{z}$ axis, the phase of such a bright spot varies along the distance from the obstacle. The phase can be calculated by considering the optical path difference (OPD) between a plane wave along the z-direction and the diffracted waves from the rim. With the disc of radius $r$ one finds the optical path as $\left(r^{2}+z^{2}\right)^{1 / 2}$ and the distance to the disc which is just $z$. We find the path difference as 


$$
O P D_{\text {planewave-diffracted wave }}=\sqrt{r^{2}+z^{2}}-z \text {. }
$$

This effect can be interpreted as Superluminal phase propagation [15]. We use the same measurement principle as for the photonic nanojet. The results are shown in Fig. 2, and the profile of the axial phase from Eq. (1) is plotted with the experimental result in Fig. 2(b) for comparison. To the best of our knowledge these are the first measurements of such phase profiles for Arago spots created with micrometer-size objects. We will give details on this measurement and discuss limitations of our method.

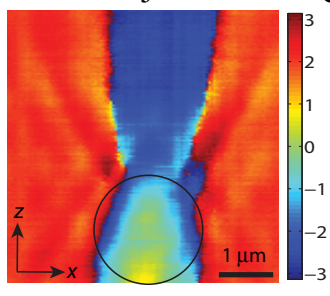

(a)

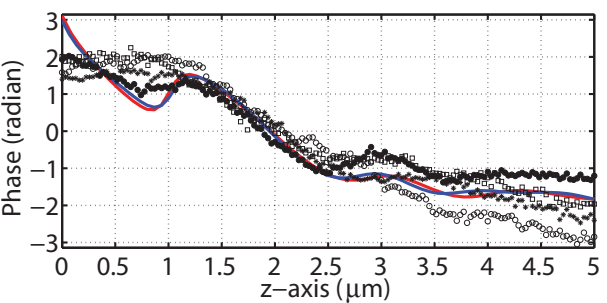

(b)

Fig. 1. (a) Measured longitudinal-differential phase distributions of the photonic nanojet spot created by a $2-\mu \mathrm{m}$ glass sphere. The dark circle indicates the $2-$ $\mu \mathrm{m}$ glass sphere. (b) Profiles of differential phase in the axial direction (z-axis) along a central line of the photonic nanojets. The solid red and blue lines correspond to the rigorous simulations for $\mathrm{NA}=0.9$ and for $\mathrm{NA}=1$, respectively, and the various markers correspond to four individual measurements. The filled circles correspond to the experimental results presented in (a). Results are from Ref. 14.

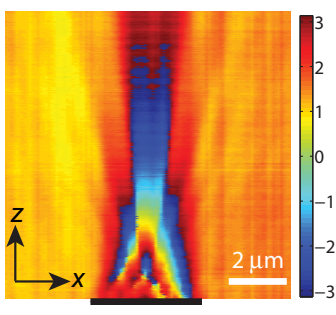

(a)

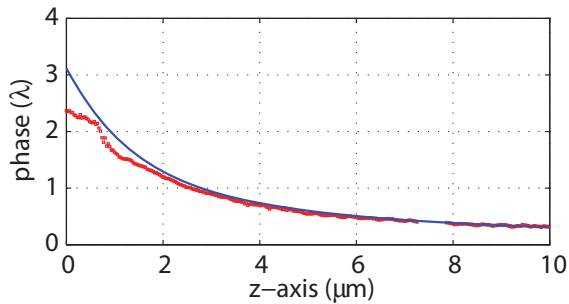

(b)

Fig. 2. (a) Measured longitudinal-differential phase distributions of the spot of Arago created by a $4-\mu \mathrm{m} \mathrm{Cr}$ disc. The dark bar at the bottom indicates the $4-$ $\mu \mathrm{m}$ Cr disc. (b) Profiles of differential phase in the axial direction (z-axis) along a central line of the circular obstacle. The solid line corresponds to the calculated data by Eq. (1)., and the open squares correspond to the measurement in Fig. 2(a).

\section{References}

[1] L. G. Gouy, "Sur une propriété nouvelle des ondes lumineuses," C. R. Hebdomadaires Séances Acad. Sci. 110, 1251 (1890).

[2] T. D. Visser and E. Wolf, "The origin of the Guoy phase anomaly and its generalization to astigmatic wavefields", Opt. Commun. 283, 3371 (2010).

[3] A. E. Siegman, Lasers (University Science books, 1986). Note that Gouy’s name is misspelled as "Guoy".

[4] P. Martelli, M. Tacca, A. Gatto, G. Moneta, and M. Martinelli, "Gouy phase shift in nondiffracting Bessel beams," Opt. Express 18, 7108 (2010).

[5] J. Hamazaki, Y. Mineta, K. Oka, and R. Morita, "Direct observation of Gouy phase shift in a propagating optical vortex," Opt. Express 14, 8382 (2006).

[6] H. Chen, Q. Zhan, Y. L. Zhang, and Y. P. Li, "The Gouy phase shift of the highly focused radially polarized beam," Phys. Lett. A 371, 259 (2007).

[7] W. Zhu, A. Agrawal, and A. Nahata, "Direct measurement of the Gouy phase shift for surface plasmon-polaritons," Opt. Express 15, 9995 (2007).

[8] I. G. da Paza, M. C. Nemes, S. Pádua, C. H. Monken, and J. G. Peixoto de Faria, "Indirect evidence for the Gouy phase for matter waves," Phys. Lett. A 374, 1660 (2010).

[9] A. Heifetz, S.-C. Kong, A. V. Sahakian, A. Taflove, and V. Backman, "Photonic Nanojets," J. Comput. Theor. Nanosci. 6, 1979 (2009).

[10] J. E. Harvey, and J. L. Forgham, “The Spot of Arago - New Relevance for an Old Phenomenon,” Am. J. Phys. 52, 243 (1984).

[11] C. Rockstuhl, I. Märki, T. Scharf, M. Salt, H. P. Herzig, and R. Dändiker, "High Resolution Interference Microscopy: A Tool for Probing Optical Waves in the Far-Field on a Nanometric Length Scale," Current Nanoscience 2, 337 (2006)

[12] M.-S. Kim, T. Scharf, and H. P. Herzig, "Small size microlenes characterization by Multiwavelength High Resolution Interference Microscopy," Opt. Express 18, 14319 (2010).

[13 ]M.-S. Kim, T. Scharf, S. Mühlig, C. Rockstuhl, and H. P. Herzig, “Engineering photonic nanojets,” Opt. Express 19, 10206 (2011).

[14] M.-S. Kim, T. Scharf, S. Mühlig, C. Rockstuhl, and H. P. Herzig, "Gouy Phase Anomaly in Photonic Nanojets," Appl. Phys. Lett. 98, 191114 (2011).

[15] M. Vasnetsov, V. Pas'ko, A. Khoroshun, V. Slyusar, and M. Soskin, "Observation of superluminal wave-front propagation at the shadow area behind an opaque disk," Opt. Lett. 32, 1830 (2007).

\section{Acknowledgements}

The research leading to these results has received funding from the European Community's Seventh Framework Programme FP7-ICT-2007-2 under grant agreement No. 224226. 